\title{
GOLAB'S THEOREM
}

CURTIS M. FULTON

J. Witkowski in [3] proved a theorem of S. Golab which gives a characterization of the sphere in $E^{3}$. In this paper a simpler proof of Golab's theorem is presented. The more direct approach involved should make the geometry simpler to visualize. First we state necessary

Definitions. (I) A curve on a surface of class $C^{1}$ is called $B$ straight if the tangent planes to the surface along the curve remain always perpendicular to a fixed direction. (II) A curve on a surface of class $C^{1}$ is called $B$-plane if the tangent planes to the surface along the curve are all parallel to a fixed direction.

We wish to prove the following

TheOREM. If every geodesic of a regular surface of class $C^{3}$ is $B$ plane but not B-straight, then the surface is part of a sphere.

PROOF (INDIRECT). According to the hypothesis of our theorem each geodesic is $B$-plane, that is there exists for each geodesic a constant unit vector $\boldsymbol{V}$ which is perpendicular to the surface normal $\boldsymbol{N}$ along the geodesic. Differentiation of $V \cdot N=0$ with respect to the arc length yields $V \cdot d_{s} N=0$ where $d_{s} N$ is not identically zero since by hypothesis the geodesic is not $B$-straight. We assume that $d_{s} N \neq 0$ at the points under discussion and the later development will show that other points need not be considered. Now for a geodesic, the principal normal $\boldsymbol{n}$ is equal to the surface normal $N$. Thus the unit tangent $t$ satisfies the relation $V \cdot t=\cos \theta=$ const which can be shown by differentiation. This means that the geodesics are helices, some fixed angle $\theta$ belonging to each geodesic. Also, at a point of a geodesic the vectors $V, t$, and $d_{s} N$ are in the tangent plane and we find for the curvature $\kappa$, which along a geodesic is the same as the normal curvature,

$$
-\kappa=t \cdot d_{s} N= \pm\left|d_{s} N\right| \sin \theta .
$$

Using the third fundamental form [2, p. 103] along a geodesic, we have

$$
d_{s} N \cdot d_{s} N=-\kappa_{1} \kappa_{2}+\left(\kappa_{1}+\kappa_{2}\right) \kappa,
$$

which allows us to change (1) to

Received by the editors November 16, 1965. 


$$
\kappa^{2}=\sin ^{2} \theta\left[-\kappa_{1} \kappa_{2}+\left(\kappa_{1}+\kappa_{2}\right) \kappa\right] .
$$

Assume the existence of a neighborhood $R_{1}$ on the surface that does not contain any umbilic points and therefore is covered by lines of curvature. Within $R_{1}$ there must be a neighborhood $R_{2}$ where the curvature $K$ does not vanish. If such an $R_{2}$ would not exist, the set of points in $R_{1}$ with $K=0$ would be dense and a continuity argument would show that $K=0$ at all points of $R_{1}$. In this case, however, we have geodesics that are $B$-straight [3, Lemma 1]. Such geodesics being ruled out by our hypothesis we now take a point $P$ in $R_{2}$. Consider the geodesic through $P$ in the principal direction corresponding to $\kappa_{1}$. Its curvature at $P$ is also $\kappa_{1}$ which we know to be different from zero. But then (2) shows that $d_{8} N \neq 0$ and consequently the arguments leading to (3) are valid. We can infer from (3) that $\sin ^{2} \theta=1$. Then along this geodesic $\sin ^{2} \theta$ will continue to equal 1 and continuity shows that the value of $\kappa$ found in (3) will be equal to $\kappa_{1}$ throughout. Hence the geodesic coincides with the line of curvature in $R_{2}$. Also, in $R_{2}$ the lines of curvature can be used as coordinate curves $[1, \mathrm{p} .56]$. Since they are geodesics, $R_{2}$ has curvature $K=0[1$, p. 45].

It follows that $K$ is identically zero in $R_{1}$. Therefore, a neighborhood $R_{1}$ without umbilics satisfying the hypothesis of our theorem cannot exist. Rather, the set of umbilics on the surface is dense and we are dealing with part of a sphere [3, Lemma 2].

\section{REFERENCES}

1. D. Laugwitz, Differential and Riemannian geometry, Academic Press, New York, 1965.

2. D. J. Struik, Lectures on classical differential geometry, 2nd ed., AddisonWesley, Reading, Mass., 1961.

3. J. Witkowski, On a characterization of the Euclidean sphere, Ann. Polon. Math. 13 (1963), 121-127.

University of California, Davis 